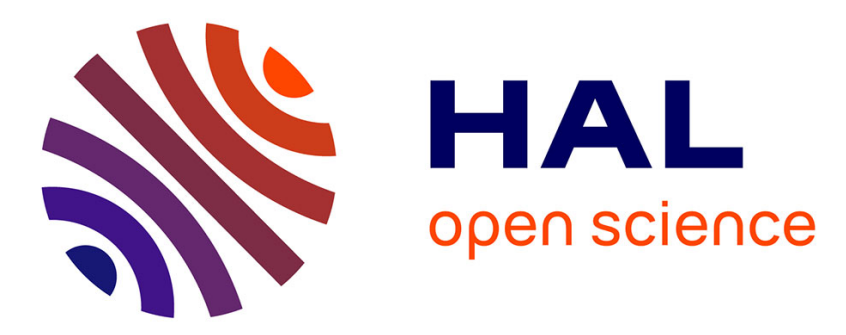

\title{
Characterization of lattice modular robots by discrete displacement groups
}

\author{
Nicolas Brener, Faïz Ben Amar, Philippe Bidaud
}

\section{To cite this version:}

Nicolas Brener, Faïz Ben Amar, Philippe Bidaud. Characterization of lattice modular robots by discrete displacement groups. IEEE/IROS Int. Conf. on Robots and Intelligents Systems, 2010, Taipei, Taiwan. pp.1133-1139. hal-03177936

\section{HAL Id: hal-03177936 https://hal.science/hal-03177936}

Submitted on 24 Mar 2021

HAL is a multi-disciplinary open access archive for the deposit and dissemination of scientific research documents, whether they are published or not. The documents may come from teaching and research institutions in France or abroad, or from public or private research centers.
L'archive ouverte pluridisciplinaire HAL, est destinée au dépôt et à la diffusion de documents scientifiques de niveau recherche, publiés ou non, émanant des établissements d'enseignement et de recherche français ou étrangers, des laboratoires publics ou privés. 
archives-ouvertes

\title{
Characterization of lattice modular robots by discrete displacement groups
}

\author{
Nicolas Brener, Faïz Ben Amar, Philippe Bidaud
}

\section{To cite this version:}

Nicolas Brener, Faïz Ben Amar, Philippe Bidaud. Characterization of lattice modular robots by discrete displacement groups. Actes de IEEE/IROS Int. Conf. on Robots and Intelligents Systems, 2010, Taipei, Taiwan. pp.1133-1139. hal-03177936

\section{HAL Id: hal-03177936 \\ https://hal.archives-ouvertes.fr/hal-03177936}

Submitted on 24 Mar 2021

HAL is a multi-disciplinary open access archive for the deposit and dissemination of scientific research documents, whether they are published or not. The documents may come from teaching and research institutions in France or abroad, or from public or private research centers.
L'archive ouverte pluridisciplinaire HAL, est destinée au dépôt et à la diffusion de documents scientifiques de niveau recherche, publiés ou non, émanant des établissements d'enseignement et de recherche français ou étrangers, des laboratoires publics ou privés. 


\title{
Characterization of Lattice Modular Robots by Discrete Displacement Groups
}

\author{
Nicolas Brener, Faiz Ben Amar, Philippe Bidaud \\ Université Pierre et Marie Curie - Paris 6 \\ Institut des Systèmes Intelligents et de Robotique, CNRS FRE 2507 \\ 4 Place Jussieu, 75252 Paris Cedex 05, France \\ Email : \{brener,amar,bidaud\}@isir.upmc.fr
}

\begin{abstract}
The paper provides a method to determine and compare the reconfigurability of lattice systems. First it shows the difference that exists between the reconfigurability and self-reconfigurability features of a lattice system. Then a method using displacement groups is introduced to characterize these features. Based on this method, these features are then compared for some existing lattice systems.
\end{abstract}

\section{INTRODUCTION}

As stated [1] lattice systems are a class of modular self-reconfigurable robots having their "modules arranged nominally in a $2 \mathrm{D}$ or $3 \mathrm{D}$ grid structure. For this category, there are discrete positions that a given module can occupy". Most lattice systems have continuous actuation mechanism [2], [3], [4], [5], [6], others use discrete [7] or passive [8] actuation. Some systems [9], [10], [11] with continuous actuation may also use both lattice and non-lattice configurations modes. In the sequel we consider only the lattice utilization, and therefore all these systems are considered as lattice ones. Only lattice systems performed successfully autonomous self-reconfiguration because the discrete configurations helps to bring the connectors to matching positions, and simplify the planning of the reconfiguration sequences. Nevertheless, the kinematical conception of lattice robots is difficult because modules with arbitrary discrete joint configurations will not necessarily lead to lattice systems; conversely, non-discrete joint configurations may lead to lattice systems because of redundant mechanisms or singular configurations. This issue was addressed in [12], [13] by proposing a framework for the kinematical design of lattice robots relying on discrete displacement groups theory. The configuration of a module $M_{i}$ is the $n$ tuple $X_{i}=\left(x_{i}^{1}, \ldots, x_{i}^{n}\right)$ where $x_{i}^{1}, \ldots, x_{i}^{n} \in \mathbb{R}^{6}$ denote the $n$ poses of its $n$ connectors $c_{i}^{1}, \ldots, c_{i}^{n}$. For a lattice system it is assumed that a module can have only a finite number of relative poses of its connectors.Any configuration of a system $S$ of $k$ modules is given by the set $\mathcal{X}=\left\{X_{1}, \ldots, X_{k}\right\}$. As stated in [12], [13], a system is said lattice if, for any configuration $\mathcal{X}$, the pose $x_{i}^{j}$ of a connector $c_{i}^{j}$ belongs to a discrete set of poses $\mathcal{O}_{i}^{j}$, called orbit, and that all the orbits $\mathcal{O}_{1 \ldots k}^{1 \ldots n}$ are generated by an unique discrete displacement group ${ }^{1} G$. Thus, for any poses $x$ and $y$ of a connector, $x$ and $y$ belongs to the same orbit and there exists $g \in G$ such that $y=g x$.

The present paper proposes an opposite approach by using discrete displacement groups to characterize the kinematical features of existing lattice robots. This allows to show that our definition is consistent with the previous one by characterizing successfully existing "lattice" systems. The first section introduces and compares the concepts of reconfiguration group and manipulation group. The second section proposes a method to establish the reconfiguration and manipulation groups for any lattice system. Based on this result, the third section describes and compares the feature of several lattice system, before concluding.

\section{Reconfiguration vs Self-Reconfiguration}

In a "Self-reconfiguration" the lattice system changes its topology by disconnecting, manipulating and reconnecting its modules. In a "reconfiguration" an external device manipulates the modules of the lattice system to reconfigure it. The external device may be, for example, a human operator, and is considered as a manipulator that can reach arbitrary poses in an unlimited workspace. The following example illustrates that some feasible reconfigurations may not be done by self-reconfigurations.

Consider the module depicted in Fig.1. It has a revolute joint with two configurations and three hermaphrodite connectors represented by the triangles.

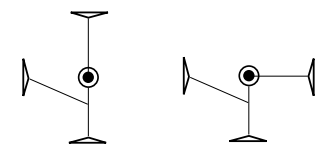

Fig. 1. A module with two joint configurations

The figure 2 represents a set of modules assembled together with a lattice represented by grey and white chessboard cells. Obviously, in a reconfiguration it is possible for an external device to exchange two modules belonging to arbitrary cells (with same color or different

\footnotetext{
${ }^{1}$ Several works about modular robots used group theory for various purposes as reconfiguration planning [14], [15] and nonisomorph assembly enumeration[16].
} 


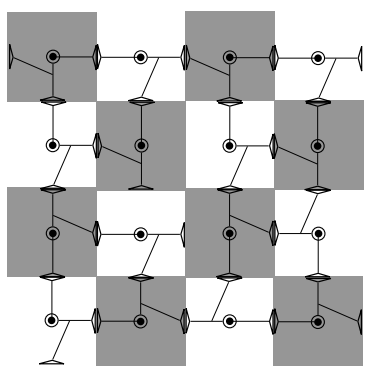

Fig. 2. Modules assembly

colors).

Nevertheless, in a self-reconfiguration, the system can displace the modules only by rotation of $90^{\circ}$ around the revolute joints located at the centers of the cells. Such displacements may only displace modules between cells of same color. Therefore a self-reconfiguration cannot exchange modules on cells with different colors. Another example: the modules of Telecube[6] can have their orientation reconfigured by hand, while a self-reconfiguration cannot change their orientation. These examples illustrate the difference between reconfiguration and selfreconfiguration capacity.

In the latter these features will be characterized respectively by the reconfiguration group and the manipulation group of a system. These groups can be determined by their generating sets. The next section explains how to construct these generating sets for lattice systems.

\section{iII. Generating Sets of the Reconfiguration GROUP AND THE MANIPULATION GROUP}

As a preliminary the terminology and symmetry operations concerning the connectors of lattice modules, introduced in [12], [13], are reminded.

\section{A. Connectors symmetries}

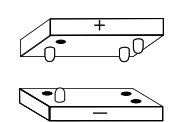

(a)

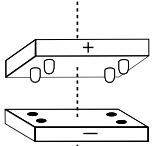

(c)

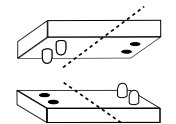

(b)

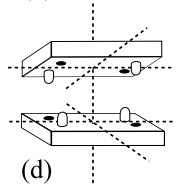

Fig. 3. Rotation axes corresponding to the symmetry operations of the connectors. (a) no symmetry. (b) transverse symmetry. (c) normal symmetry. (d) transverse and normal symmetries.

1) A connector without symmetry has no symmetry axis. It can be connected to a connector with opposite gender. Fig. 3(a) represents two compatible connectors with genders + and - .
2) A connector may have a "transverse axis" 2 " corresponding to a 2 -fold rotation axis as represented Fig. 3(b). Such a connector is said "hermaphrodite" and can be connected to another one identical to itself (the two connectors in Fig. 3(b) are identical).

3) A connector may have a "normal symmetry" corresponding to a $n$-fold rotation axis as represented Fig. 3(c). The order $n$ of the rotation equals the number of orientations the connector can be connected to another one having an opposite gender. In the example Fig. 3(c) the normal symmetry axis of the connector (considered rectangular) is a 2 fold rotation axis, therefore the connector + can be connected with 2 different orientations to the connector - (if the connector is considered square the order of the rotation is 4 and it has 4 connecting orientations).

4) A connector may have both types of symmetries described previously: it has one normal symmetry axis of order $n$, and $n$ transverse symmetry axes of order 2. For instance, Fig. 3(d) represents a connector with one normal symmetry of order 2 and two transverse axis with order 2 . Such a connector is hermaphrodite and can be connected to another one identical to itself.

Terminologically, we call configuration of a connector the set of points that it occupies in the space. Therefore several poses can correspond to the same configuration because of a normal symmetry axis of the connector. Two compatible connectors with opposite genders + and - are called "opposite connectors". When two such connectors are connected (or have configurations such that they can be connected) we say that they have "opposite configurations". Connectors which are compatible (hermaphrodite or with opposite gender) or identical (hermaphrodite or with same gender) are said of "same type".

\section{B. Geometrical features}

We can now describe the geometrical features of the modules that will be used to construct the generating sets of the reconfiguration group and the manipulation group. There are three types of displacements that must be taken in account:

1) The first type of displacement corresponds to the symmetry operations of the connectors of a module as recalled section III-A. For instance, the Fig.4(a) represents a configuration of a module of the system M-Tran[10] which has 6 connectors. The symmetry operations of the connectors are the $90^{\circ}$ rotation around their normal axes represented by dotted lines.

2) The second type of displacement will be called inter-connectors displacement. It can correspond to:

${ }^{2}$ Called "tangential axis" in [12], [13] 
a) a displacement that brings a connector of a module to the same configuration as another identical connector of the module. For instance, in Fig.4(b) the connector A and B are identical and the $90^{\circ}$ rotations around the dotted line brings the connector A to the same configuration as the connector B.

b) a displacement that brings a connector to a configuration such that it could be connected to an opposite connector of the module (the connectors reach opposite configurations). Such a displacement exists only for non-hermaphrodite connectors. For example, in Fig.4(c) the connectors B and $\mathrm{C}$ are opposite and the $180^{\circ}$ rotation around the dotted line move the connector $\mathrm{B}$ at a configuration opposite to the configuration of the connector C.

3) The third type of displacement will be called interconfigurations displacement. Is is a displacement of a connector of a module from one pose to a new pose when another connector of the same module is assumed immobile. Such displacements correspond to the relative displacements of connectors of a module due to its actuation mechanisms. If the joints of the module are independent (this is mostly the case), any motion produced by one joint corresponds to an inter-configuration displacements. For instance, Fig.4(d) shows the $90^{\circ}$ rotation axes of displacements produced by the joints M1 and M2. For redundant mechanism, parallel mechanism, singular configurations, or coupled joints[17], it is not possible to assimilate joints motions with connectors displacements.

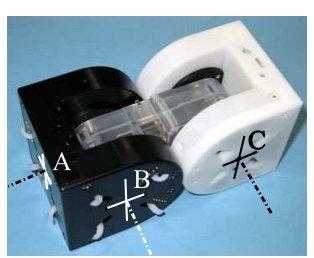

(a) Connectors symmetries

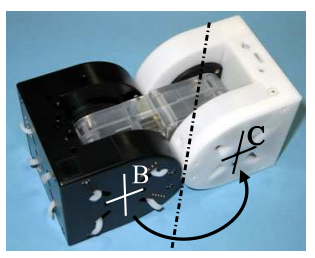

(c) Inter-connectors displacement (opposite connector)

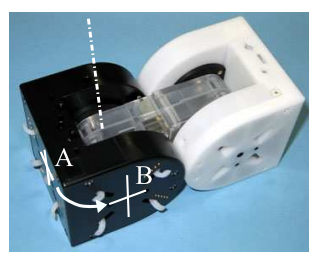

(b) Inter-connectors displacement (identical connector)

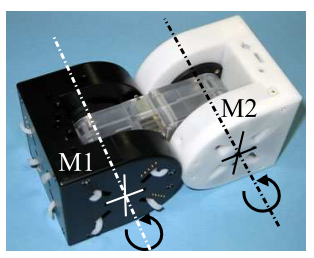

(d) Inter-configurations displacements
Fig. 4. Symmetry elements of the module M-Tran[10].

\section{Reconfiguration group}

Consider one module $M$ having $n$ connectors $c_{1}, \ldots, c_{n}$ with a constant configuration $X=\left(x_{1}, \ldots, x_{n}\right)$. We denote by $\Omega \subset S E(3)$ the set containing all the symmetry operations of the connectors of the module, by $\Delta \subset S E(3)$ the set of inter-connectors displacement of the module, and by $\Gamma \subset S E(3)$ the set of the interconfigurations displacements from the configuration $X$ of the module to any other configuration $X^{\prime}=\left(x_{1}^{\prime}, \ldots, x_{n}^{\prime}\right)$ such that at least one connector keeps the same pose: for any $X^{\prime}$ there exists $i$ such that $x_{i}^{\prime}=x_{i}$. Moreover it is assumed (1) that the module has finitely many relative poses of its connectors, (2) that it has finitely many connectors and (3) that the number of symmetry operations for each connector is finite, so $\Gamma, \Delta$ and $\Omega$ are finite sets.

Definition 1 (Reconfiguration group): The reconfiguration group of the (homogeneous) modular system defined by the module $M$ is the group generated by the set $S=\Omega \cup \Delta \cup \Gamma$ with configuration $X=\left(x_{1}, \ldots, x_{n}\right)$. Moreover, if the group generated by $S$ is a discrete displacement group, we say that the modular system is lattice, otherwise it is not a lattice system.

Definition 2 (Group type): Two displacements groups $G \subseteq S E(3)$ and $G^{\prime} \subseteq S E(3)$ are of same type if they are conjugate by a displacement ${ }^{3}: \exists h \in S E(3)$ such that $g \in G$ iff $h g h^{-1} \in G^{\prime}$.

Theorem 1: The type of the reconfiguration group $G$ of the module $M$ does not depends on the configuration $X=\left(x_{1}, \ldots, x_{n}\right)$ of $M$.

Proof: $X$ can be defined by the 2-tuple $\left(x_{1}, D\right)$ where $D \in S E(3)^{n-1}$ is a vector of displacements between $x_{1}$ and each $n-1$ other connectors (so $\Delta \subseteq D$ ).

First, changing the relative configuration of the connectors of the module $M$ by letting the connector $c_{1}$ unmoved, leads to configuration $X^{\prime}=\left(x_{1}, D^{\prime}\right)$. Let $x_{2}^{\prime}, \ldots, x_{n}^{\prime}$ denote the poses of $c_{2}, \ldots, c_{n}$, and $G^{\prime}, S^{\prime}, \Omega^{\prime}, \Delta^{\prime}, \Gamma^{\prime}$ denote the new $G, S, \Omega, \Delta, \Gamma$. For any $x_{i}^{\prime} \neq x_{i}$, we have $x_{i}^{\prime}=\gamma x_{i}$ where $\gamma \in \Gamma$, aka for any $x_{i}^{\prime}$, $x_{i}^{\prime}=\gamma x_{i}$ where $\gamma \in \Gamma \cup\{1\}$. Therefore, the connectors symmetries are conjugated by elements of $\Gamma \cup\{1\}$ : for any $\omega^{\prime} \in \Omega^{\prime}, \omega^{\prime}=\gamma \omega \gamma^{-1}$ where $\omega \in \Omega$, thus the elements of $\Omega^{\prime}$ are products of elements of $\Omega \cup \Gamma$ and their inverses, so $\Omega^{\prime}$ is included in the group generated by $\Omega \cup \Gamma$. Since $G$ is generated by $\Omega \cup \Delta \cup \Gamma$, we have $\Omega^{\prime} \subset G$.

Similarly, any inter-configuration displacement $\gamma_{1}$ with respect to connector $c_{i}$ with pose $x_{i}^{\prime}$ is that with respect to $c_{i}$ with pose $x_{i}$ conjugated by $\gamma \in \Gamma \cup\{1\}$ such that $x_{i}^{\prime}=\gamma x_{i}$ : for any $\gamma_{1} \in \Gamma^{\prime}, \gamma_{1}=\gamma \gamma_{0} \gamma^{-1}$ where $\gamma_{0} \in \Gamma$. Therefore the elements of $\Gamma^{\prime}$ are products of elements of $\Gamma$ and their inverses, thus $\Gamma^{\prime} \subset G$.

For any displacement between connectors with poses $x_{i}^{\prime}$ and $x_{j}^{\prime}$ we have $d_{x_{i}^{\prime} x_{j}^{\prime}}=d_{x_{j} x_{j}^{\prime}} d_{x_{i} x_{j}} d_{x_{i}^{\prime} x_{i}}$ so for any $\delta_{i j} \in$ $\Delta, \delta_{i j}^{\prime} \in \Delta^{\prime}, \delta_{i j}^{\prime}=\gamma_{1} \delta_{i j} \gamma_{2}^{-1}$ where $\gamma_{1}, \gamma_{2} \in \Gamma \cup\{1\}$, thus

\footnotetext{
${ }^{3}$ In crystallography a similar definition[18] considers an isometry instead of a displacement.
} 
the elements of $\Delta^{\prime}$ are products of elements of $\Delta \cup \Gamma \cup\{1\}$ and their inverses, so $\Delta^{\prime} \subset G$.

Thereby $\Omega^{\prime} \cup \Delta^{\prime} \cup \Gamma^{\prime} \subset G$ aka $S^{\prime} \subset G$, hence $G^{\prime} \subseteq G$. Since $X=\left(x_{1}, D\right)$ and $X^{\prime}=\left(x_{1}, D^{\prime}\right)$ are arbitrary, considering $X=\left(x_{1}, D^{\prime}\right)$ and $X^{\prime}=\left(x_{1}, D\right)$ leads to $G^{\prime} \supseteq G$, therefore $G^{\prime}=G$.

$\underline{\text { Second, }}$, consider the new configuration $Y=\left(y_{1}, \ldots, y_{n}\right)$ such that all the connectors of the module $M$ has been moved by $d \in S E(3)$, leaving the relative configuration of the connectors unchanged. Let denote $G^{\prime}$ and $S^{\prime}$ the new $G$ and $S$.

Any element $b$ (resp. $b^{\prime}$ ) of $G$ (resp. $G^{\prime}$ ) is a product of elements $a_{1}, a_{2}, \ldots a_{n}$ (resp. $a_{1}^{\prime}, a_{2}^{\prime}, \ldots a_{n}^{\prime}$ ) of $S$ (resp. $\left.S^{\prime}\right)$ and their inverses: $b=a_{1} a_{2} \ldots a_{n}, b^{\prime}=a_{1}^{\prime} a_{2}^{\prime} \ldots a_{n}^{\prime}$.

Because of the displacement $d$ of the module $M$ any element $a^{\prime}$ of $S^{\prime}$ is an element $a$ of $S$ conjugated by $d$ : $a^{\prime}=d a d^{-1}$. Therefore $b^{\prime} \in G^{\prime} \Leftrightarrow b^{\prime}=a_{1}^{\prime} a_{2}^{\prime} \ldots a_{n}^{\prime} \Leftrightarrow b^{\prime}=$ $d a_{1} d^{-1} d a_{2} d^{-1} \ldots d a_{n} d^{-1} \Leftrightarrow b^{\prime}=d a_{1} a_{2} \ldots a_{n} d^{-1} \Leftrightarrow b^{\prime}=$ $d b d^{-1}$, where $b \in G$. Thus $G$ and $G^{\prime}$ are identical up to a conjugacy by a displacement.

For instance, for the module M-Tran[10] Fig.4, $S$ contains 4 symmetries for each 6 connectors, plus 5 inter-connectors displacement for each 6 connectors, plus 8 inter-configurations for when the connectors + are assumed immobile and 8 other inter-configurations when the connectors - are assumed immobile, providing $24+$ $30+16=70$ generators.

1) Simplification: By definition $S$ is a generating set of the reconfiguration group, but any subset of $S$ which generates the same group as $S$ may be used instead of $S$. Considering a module $M$ with a constant configuration $X$, a reduced generating set, denoted $\bar{S}$ can consist of:

1) for each type of connector, the symmetry operations of only one connector (chosen arbitrarily) of this type;

2) for each type of connector, the inter-connectors displacements from only one connector (chosen arbitrarily) to the others of same type;

3) the inter-configurations displacements from the configuration $X$ to the others, when only one connector, chosen arbitrarily, is assumed immobile.

Proof: Let denote $\bar{S}=\bar{\Omega} \cup \bar{\Delta} \cup \bar{\Gamma}$ the reduced sets, and $\langle\bar{S}\rangle,\langle\bar{\Omega}\rangle,\langle\bar{\Delta}\rangle,\langle\bar{\Gamma}\rangle$ the generated groups.

For any type of connector, $\bar{\Delta}$ contains the displacement $\delta_{k i}$ from one (chosen) connector $k$ of this type to any other connector $i$ of the same type. For any displacement between two connectors $i, j$ of same type $\delta_{i j}=\delta_{k j} \delta_{i k}$, $\delta_{k j} \delta_{i k}^{-1} \in \bar{\Delta}$ so the elements of $\Delta$ are product of elements of $\bar{\Delta}$ and their inverses, thus:

$$
\Delta \subset\langle\bar{\Delta}\rangle
$$

For any connector symmetry $\omega \in \Omega, \omega=\delta \omega^{\prime} \delta^{-1}$ where $\omega^{\prime} \in \bar{\Omega}$ and $\delta^{\prime} \in \Delta$, thus $\Omega \subset\langle\Delta\rangle \cup\langle\bar{\Omega}\rangle$. Moreover (1) $\Rightarrow$ $\langle\Delta\rangle \subseteq\langle\bar{\Delta}\rangle$, so:

$$
\Omega \subset\langle\bar{\Delta}\rangle \cup\langle\bar{\Omega}\rangle
$$

Let denote $d_{x_{i} y_{i}} \in \Gamma$ the displacement of the connector $c_{i}$ between two configurations $X=\left(x_{1}, \ldots, x_{k}, \ldots, x_{l}, \ldots, x_{i}, \ldots, x_{n}\right)$ and $Y=\left(y_{1}, \ldots, x_{k}, \ldots, y_{l}, \ldots, y_{i}, \ldots, y_{n}\right) \quad\left(c_{k} \quad\right.$ is immobile) and $d_{x_{i} z_{i}} \in \Gamma$ the displacement of $c_{i}$ between the configurations $X$ and $Z=$ $\left(z_{1}, \ldots, z_{k}, \ldots, x_{l}, \ldots, z_{i}, \ldots, z_{n}\right)$ (here $c_{l}$ is immobile). We have $d_{x_{i} z_{i}}=d_{y_{i} z_{i}} d_{x_{i} y_{i}}$. Moreover, we assume that $Y$ and $Z$ correspond to the same relative configuration of the connectors, therefore $d_{y_{i} z_{i}}=d_{y_{l} x_{l}}$, thus $d_{x_{i} z_{i}}=d_{y_{l} x_{l}} d_{x_{i} y_{i}}$. We assume also that $\bar{\Gamma}$ contains the inter-configurations displacements with the connector $c_{k}$ having a constant configuration $x_{k}$, therefore $d_{x_{i} y_{i}} \in \bar{\Gamma}$ and $d_{y_{l} x_{l}}^{-1} \in \bar{\Gamma}$, so $d_{x_{i} z_{i}}$ equals a product of elements of $\bar{\Gamma}$ and their inverses, thus $d_{x_{i} z_{i}} \in\langle\bar{\Gamma}\rangle$. Since the relative configuration defined by $Y$ and $Z$ is arbitrary, any other inter-configurations displacement of $\Gamma$ with $c_{l}$ immobile can be expressed as a product of elements of $\bar{\Gamma}$ and their inverses. Moreover $c_{l}$ is arbitrary, so any inter-configurations displacement with any immobile connector is a product of elements of $\bar{\Gamma}$ and their inverses, thus:

$$
\Gamma \subset\langle\bar{\Gamma}\rangle
$$

(1), (2), (3) $\Rightarrow \Omega \cup \Delta \cup \Gamma \subset\langle\bar{\Omega}\rangle \cup\langle\bar{\Delta}\rangle \cup\langle\bar{\Gamma}\rangle \Rightarrow S \subset\langle\bar{S}\rangle \Rightarrow$ $\langle S\rangle \subseteq\langle\bar{S}\rangle$. Moreover $S \supset \bar{S} \Rightarrow\langle S\rangle \supseteq\langle\bar{S}\rangle$ so $\langle S\rangle=\langle\bar{S}\rangle$ aka $G=\langle\bar{S}\rangle$

For the module M-Tran[10] Fig.4, $\bar{S}$ contains the 4 symmetries of 1 connector (there is one type of connector), 5 inter-connectors displacements from one connector (chosen arbitrarily) to the others, 8 inter-connectors displacements from one configuration to the others when a connector is immobile, providing $4+5+8=17$ generators.

2) Further simplifications: If the joints of a module are not coupled (which is the case for most lattice system, an exception is [17]) a further simplification is to put in $\bar{\Gamma}$ the sets of inter-configurations displacements produced by each joint separately, because the other interconfigurations displacements will result as combinations of the previous ones. For the module M-Tran this leads to consider only 3 displacements for one joint and 3 for the other.

We have see that $S$ is redundant because the smaller set $\bar{S}$ generates the same group. Nevertheless, in most cases, the generating set $\bar{S}$ his itself redundant because its displacements occur along common symmetry axes. Therefore it may be advantageous to represent the generating set $\bar{S}$ geometrically by using symmetry axes. In the following example we will see that the generating set $\bar{S}$ of M-Tran Fig.4 can be represented by four 4 -fold rotation axes and one translation.

3) Example: The kinematics of the system M-Tran[10] is represented on the Fig.5. It has two revolute joints axes M1 and M2, and 6 connectors: three connectors C1, C2 and $\mathrm{C} 3$ with gender + and three opposite connectors C4, C5 and C6 with gender -. 


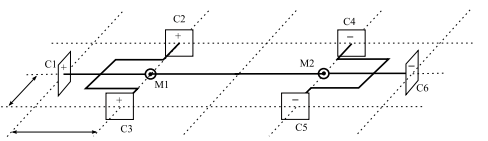

Fig. 5. The kinematics of a module M-Tran.

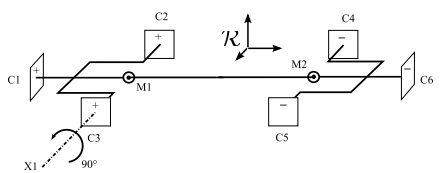

(a) Connectors symmetries

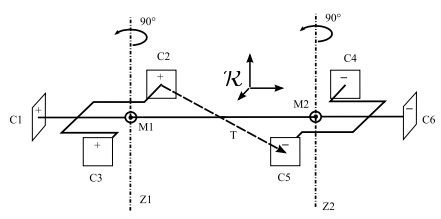

(b)

Inter-connectors

displacements

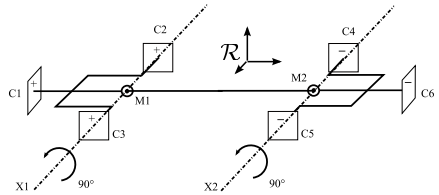

(c) Inter-configurations displacements

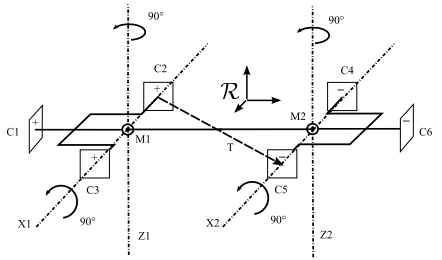

(d) Resulting symmetry axes

Fig. 6. Symmetry axes of the module M-Tran[10].

The displacements of $\bar{S}$ (see section III-C.1) are represented geometrically by symmetry axes:

1) Fig. 6(a) shows a 4-fold rotation axis corresponding to the symmetry operations of one connector.

2) Fig. 6(b) shows the symmetry axes corresponding to the inter-connectors displacements. The rotations around the 4 -fold axis $\mathrm{Z} 1$ provides the displacements to bring the connector $\mathrm{C} 2$ to the same configurations as the identical connectors $\mathrm{C} 1$ and C3. Moreover, the translation along the axis $\mathrm{T}$ and the rotations along the 4-fold axis Z2 allows to bring the connector $\mathrm{C} 2$ on the opposite connectors C4, C5 and C6.

3) Fig. 6(c) shows the 4-fold rotation axes corresponding to the displacements generated by the joints M1 and M2.

The resulting set of axes is recapitulated Fig. 6(d).
4) Recognizing the generated reconfiguration group: The chiral space groups ${ }^{4}$ are all the discrete displacement groups containing translations. Therefore a modular system is lattice and has a translational periodicity in the space if and only if its reconfiguration group is a chiral space group. To find the corresponding space group, one must find the minimum group generated by $\bar{S}$ in the tables [18], [19] or find the minimum group whose symmetry axes represented in [18], [20] match the geometrical representation of $\bar{S}$ (as in Fig. 6(d)). If $\bar{S}$ does not generate a chiral space group, the system is not lattice or has no periodicity in three directions (for example, we consider the Caisson Fig. 35 in [17] as a lattice system with a periodicity in only one direction so its reconfiguration group is not a chiral space group). For the

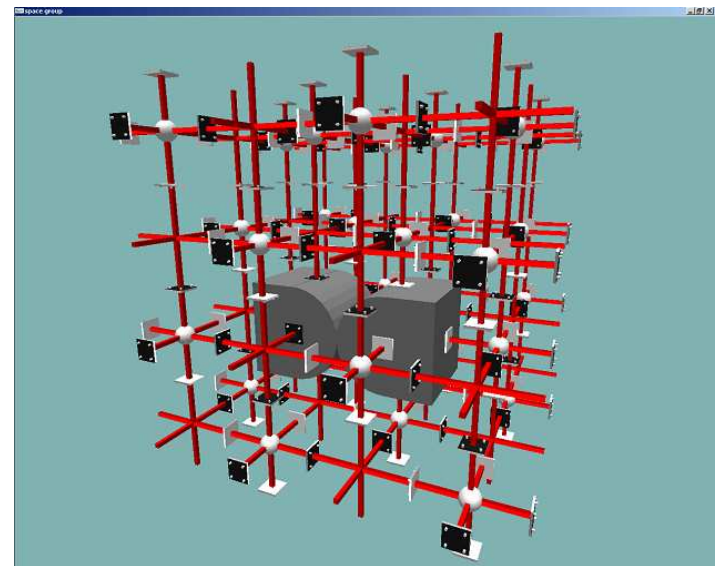

Fig. 7. The module M-Tran and a representation of the 4-fold rotation axes of its corresponding reconfiguration group.

system M-Tran, $G$ is the face centered space group F432. The Fig.7 represents the 4-fold rotation axes (Wyckoff position $e$, see [18]) of the space group F432 and a module of the system M-Tran. The white spheres represent the position equivalent by translation. The 4 -fold rotation axes of F432 coincide with the 4-fold symmetry axes of the connectors and with the 4 -fold rotation axes of the revolute joints in any possible lattice configuration of the module. All the "equivalent" poses of a connector can be obtained by applying all the displacements of the group to one pose of this connector. On the figure, all the equivalent poses of the connectors + and - are represented respectively by the black and white squares (poses corresponding to the same connector configuration are indistinguishable because of its normal symmetry). In any lattice configuration of the system, the connectors of

\footnotetext{
${ }^{4}$ The chiral space groups (also called Sohncke groups) where introduced to describe the (chiral) crystals symmetry because they contains three independent translations allowing translational periodicity in the space. There exists 65 types of such groups described in [18] and listed in [12]. In two dimensions the discrete groups having two independent translations are called the plane groups. The 17 types of plane groups are described in [18]. Exhaustive data about the 230 space groups and their hierarchy can be found on line in [19], [20].
} 
the modules will coincide with the equivalent poses of these connectors.

\section{Manipulation group}

The manipulation group is the minimum group containing the displacements that the actuation mechanisms of the modules may produce in any configuration of the modules. Let $\mathcal{D}$ be the set (assumed finite) of displacements that a mechanism of a module $M$ can produce when a connector $c$ of $M$ has a constant pose. Moving $c$ (and consequently $M$ ) by a displacement $d \in S E(3)$ brings $c$ to a new constant pose. The new set of displacement produced by the mechanism is the previous one conjugated by $d$ :

$$
\mathcal{D}^{\prime}=d \mathcal{D} d^{-1}
$$

The set $\mathcal{D}$ corresponds to the inter-configurations displacements $\bar{\Gamma}$ of the module, and the displacement $d$ may be any element of the reconfiguration group $\mathcal{R}$ generated by $\bar{S}=\bar{\Omega} \cup \bar{\Delta} \cup \bar{\Gamma}$.

Hence, the manipulation group $\mathcal{M}$ is the smallest group containing the inter-configurations displacements of the module conjugated by the elements of its reconfiguration group:

$$
\mathcal{M}=\left\langle g d g^{-1} \mid g \in \mathcal{R}, d \in \bar{\Gamma}\right\rangle
$$

Or, more in detail:

$$
\mathcal{M}=\left\langle g d g^{-1} \mid g \in\langle\bar{\Omega} \cup \bar{\Delta} \cup \bar{\Gamma}\rangle, d \in \bar{\Gamma}\right\rangle
$$

For any element $m$ in $\mathcal{M}, m=$ $g d_{1} g^{-1} g d_{2} g^{-1} \ldots g d_{n} g^{-1}=g d_{1} d_{2} d_{n} g^{-1}=g d g^{-1}$ where $d$ is an element of the group $\langle\bar{\Gamma}\rangle$ generated by $\bar{\Gamma}$. Therefore, $\mathcal{M}$ is the normalizer of $\langle\bar{\Gamma}\rangle$ in $\mathcal{R}$ :

$$
\mathcal{M}=\left\langle g d g^{-1} \mid g \in \mathcal{R}, d \in\langle\bar{\Gamma}\rangle\right\rangle=\operatorname{Norm}_{\mathcal{R}}\langle\bar{\Gamma}\rangle
$$

\section{E. Heterogeneous system}

In the presented method, the modular systems had only one type of module. To generalize to heterogeneous systems with $n$ types of modules one must consider $n$ modules connected together, this assembly forms a "meta-module". The generating sets are determined on the meta-module in the same manner as for one module of an homogeneous system, however the connectors linking adjacent modules of the meta-module must be considered as any other connector.

\section{Comparison of Lattice Systems}

The previous principles where used to compare the reconfiguration group and manipulation group of several existing lattice systems. The results are represented in Table I. For each system the table give the types of the reconfiguration group and manipulation group in Hermann-Mauguin notation. For these systems, the groups are 2 or 3 dimensional chiral space groups, described in [18]. The groups in lowercase ( $p 1$ or $p 4)$ are two dimensional space groups, while those in uppercase (like P432) are three dimensional space groups. The manipulation groups are necessarily subgroups of the reconfiguration groups, but may be equals ("The same" is displayed in the last column). If the manipulation group is not the same, it may nevertheless have the same type. This occurs for the system Micro-unit: in the corresponding row the scale difference is displayed. If the manipulation and reconfiguration groups have different types, then the reconfiguration group is a maximum subgroup of the manipulation group (the contrary does not occur for the existing lattice systems). For the system Stochatic[8] there is no manipulation group, because the system uses only external devices to be reconfigured.

The symmetries of the connector are also displayed. To note the symmetry of a connector (see [12], [13]) we use two digits: the first digit gives the order of the normal symmetry, while the second gives the order of the transverse symmetry (which is 1 or 2). For non hermaphrodite connectors, the transverse symmetry order equals 1, and it is possible to distinguish their gender by replacing the digit 1 by + or - . To locate the symmetry axes of the connectors in relation with the reconfiguration group (not the manipulation group) the table gives the Wyckoff positions (see [18], [12], [13] of these axes. Likewise, the table locates the joints axes in relation with the reconfiguration group by giving their corresponding Wyckoff positions. For the heterogeneous system I-Cube, the Wyckoff positions of the axes are given separately for each modules, while for the system Molecule V2 both module are described in the same row (only the gender of the connectors changes).

When the reconfiguration and manipulation groups are different this means that some identical connectors of modules cannot have their configurations exchanged by self-reconfiguration (while this is possible by reconfiguration). For example, the system Atron[9] has its modules equipped with two pair of connectors + and connectors -, but it cannot exchange the configurations of two identical connectors of a module by self-reconfiguration. Another example is the system Fracta[3], for which it is not possible to exchange the configurations of two adjacent modules by self-reconfiguration.

\section{Concluding Remarks}

The introduced method allowed to characterize successfully the existing lattice systems by discrete displacement groups. This prove that our definition of lattice systems is consistent with the former one, and consequently that the conception approach introduced in [12], [13] is appropriate for the design of lattice systems.

Moreover, this characterization helps to estimate the kinematical features of the lattice systems by giving the sets of possible displacement occurring on the module during reconfigurations or self-reconfigurations. For example if the manipulation group is the identity, the system cannot self-reconfigure. If the manipulation group is two dimensional, while the reconfiguration group has 
TABLE I

Comparison of the kinematical features of SOME LatTice Systems.

\begin{tabular}{|c|c|c|c|c|c|c|}
\hline \multirow[b]{2}{*}{ System } & \multirow{2}{*}{$\begin{array}{l}\text { Reconfiguration } \\
\text { group }\end{array}$} & \multicolumn{2}{|c|}{ Connectors } & \multicolumn{2}{|l|}{ Joints } & \multirow[b]{2}{*}{ Manipulation group } \\
\hline & & Symmetry & $\begin{array}{l}\text { Wyckoff } \\
\text { Position }\end{array}$ & Type and number & $\begin{array}{l}\text { Wyckoff } \\
\text { position }\end{array}$ & \\
\hline Atron & P432 & 11 & $\mathrm{k}$ (geneneral position) & 4-fold revolute joint: 1 & $\mathrm{e}$ & $\mathrm{F} 432$ \\
\hline Crystalline & $p 1$ (2D) & 11 & a & Prismatic joints: 4 & $\begin{array}{c}\text { a (gencral } \\
\text { position) }\end{array}$ & The same \\
\hline Fracta 3D & P432 & 22 & $\mathrm{~d}$ & 4-fold revolute joints: 6 & c & $\mathrm{F} 432$ \\
\hline I-Cube & P432 & $\begin{array}{l}\text { Cube: } 4- \\
\text { Link: } 4+\end{array}$ & e & $\begin{array}{l}\text { Cube: none } \\
\text { Link: 4-fold revolute joint: } 3\end{array}$ & $\begin{array}{c}\text { Cube: N.A. } \\
\text { Link: } e, f\end{array}$ & The same \\
\hline Micro Unit & $p 4$ (2D) & 11 & $\mathrm{~d}$ & 4 -fold revolute joints: 2 & $\mathrm{a}$ & Scale change: $x \sqrt{2}$ \\
\hline Molecube & P432 & 42 & $\mathrm{c}$ & \begin{tabular}{|l} 
3-fold rotation joints: 1 \\
\end{tabular} & $\mathrm{~g}$ & $\mathrm{~F} 23$ \\
\hline Molecule v1 & P432 & 42 & d & 4-fold rotation joints: 4 & c & $\mathrm{F} 432$ \\
\hline Molecule v2 & $\mathrm{F} 432$ & $4+, 4-$ & $\mathrm{e}$ & 4-fold rotation joints: 4 & $\mathrm{e}$ & The same \\
\hline M-tran & F432 & $4+, 4-$ & $\mathrm{e}$ & 4-fold rotation joints: 2 & $\mathrm{e}$ & The same \\
\hline Stochastic & $\mathrm{P} 432$ & 42 & c & None & - & - \\
\hline SuperBot & P432 & 42 & e & 4-fold rotation joints: 3 & e & $\mathrm{F} 432$ \\
\hline Telecube & P23 & 22 & f & Prismatic joints: 6 & $\begin{array}{l}j \text { (general } \\
\text { position) }\end{array}$ & P1 \\
\hline
\end{tabular}

three dimensions, the system can only move its modules along a plane. These results can be used as a complement for the conception of lattice systems because it allows to discriminate between candidate solutions.

Furthermore, our method can be used to analyse the effect of some modifications of lattice systems. For example, if we put hermaphrodite connectors for the modules of M-Tran[10] we will find that the new system has the same reconfiguration and self-reconfiguration groups than the previous one. If we alternate passive and active modules for the system Fracta[3] we will find that the new system keeps the same self-reconfiguration group. The Table I shows that nearly all 3D systems correspond to groups of type P432 and F432 which have respectively a cubic and cubic face centered lattice. These groups are on the top of the chiral space groups hierarchy. The reason to this is that only these groups have orthogonal 4-fold rotation axes, and most lattice systems have orthogonal $90^{\circ}$ rotations in the generating sets of their manipulation or reconfiguration groups. Nevertheless, it is surprising that no lattice system corresponds to the group of type I432 with a cubic centered lattice.

\section{REFERENCES}

[1] M. Yim, P. J. White, M. Park, and J. Sastra, "Modular selfreconfigurable robots," in Encyclopedia of Complexity and Systems Science, R. A. Meyers, Ed. Springer, 2009, pp. 56185631.

[2] D. Rus and M. Vona, "Crystalline robots: Self-reconfiguration with compressible unit modules," Autonomous Robots, vol. 10 (1), pp. 107-124, 2001.

[3] H. Kurokawa, S. Murata, E. Yoshida, K. Tomita, and S. Kokaji, "A 3-d self-reconfigurable structure and experiments," in Proc. of the 1998 IEEE Int. Conf. on Intelligent Robots and Systems, vol. 2, Victoria, BC, Canada, Oct. 1998, pp. 860-865.

[4] C. Unsal and P. Khosla, "Mechatronic design of a modular self-reconfiguring robotic system," in Proc. of the 2000 IEEE Int. Conf. on Robotics and Automation, vol. 2, San Francisco, CA, USA, Apr. 2000, pp. 1742-1747.

[5] K. Kotay and D. Rus, "Locomotion versatility through selfreconfiguration," Robotics and Autonomous Systems, vol. 26, pp. 217-232, 1999.
[6] J. W. Suh, S. B. Homans, and M. H. Yim, "Telecubes: Mechanical design of a module for self-reconfigurable robotics," in Proc. of the 2002 IEEE Int. Conf. on Robotics and Automation, vol. 4, Washington, DC, USA, May 2002, pp. 4095-4101.

[7] E. Yoshida, S. Murata, S. Kokaji, K. Tomita, and H. Kurokawa, "Micro self-reconfigurable modular robot using shape memory alloy," Journal of Robotics and Mechatronics, vol. 13 (2), pp. 212-219, 2001.

[8] P. White, V. Zykov, J. Bongard, and H. Lipson, "Three dimensional stochastic reconfiguration of modular robots," in Proc. of Robotics Science and Systems. MIT Press, 2005, pp. 161168.

[9] M. W. Jorgensen, E. H. Ostergaard, and H. H. Lund, "Modular atron: Modules for a self-reconfigurable robot," in Proc. of the 2004 IEEE/RSJ Int. Conf. on Intelligent Robots and Systems, Sendai, Japan, Sep. 2004.

[10] S. Murata, E. Yoshida, A. Kamimura, H. Kurokawa, K. Tomita, and S. Kokaji, "M-tran: Self-reconfigurable modular robotic system," IEEE/ASME Trans. on Mechatronics, vol. 7 (4), pp. 431-441, 2002.

[11] V. Zykov, E. Mytilinaios, B. Adams, and H. Lipson, "Selfreproducing machines," Nature, vol. 435, pp. 212-219, 2005.

[12] N. Brener, F. Ben Amar, and P. Bidaud, "Designing Modular Lattice Systems with Chiral Space Groups," The International Journal of Robotics Research, vol. 27, no. 3-4, pp. 279-297, 2008. [Online]. Available: http://ijr.sagepub.com/cgi/content/abstract/27/3-4/279

[13] N. Brener, F. Ben-Amar, and P. Bidaud, "From crystals to lattice robots," in Proc. of the 2008 IEEE Int. Conf. on Robotics and Automation, Pasadena, CA, USA, May 2008, pp. 3514-3519.

[14] A. Pamecha, I. Ebert-Uphoff, and G. Chirikjian, "Useful metrics for modular robot motion planning," IEEE Trans. on Robotics and Automation, vol. 13 (4), pp. 531-545, 1997.

[15] A. Abrams and R. Ghrist, "State complexes for metamorphic robots," CoRR, vol. cs.RO/0307004, 2003.

[16] I.-M. Chen and J. W. Burdick, "Enumerating the nonisomorphic assembly configurations of modular robotic systems," The Int. Journal of Robotics Research, vol. 17(7), pp. 702-719, 1998.

[17] Y. Terada and S. Murata, "Automatic modular assembly system and its distributed control," The International Journal of Robotics Research, vol. 27, no. 3-4, pp. 445-462, 2008.

[18] Space Group Symmetry, ser. International Tables for Crystallography. Kluwer Academic Publishers, 2006, vol. A.

[19] "Bilbao crystallographic server," http://www.cryst.ehu.es, 2009.

[20] "A hypertext book of crystallographic space group diagrams and tables," http://img.chem.ucl.ac.uk/sgp/mainmenu.htm, 2009. 